\title{
МАТЕМАТИЧЕСКОЕ МОДЕЛИРОВАНИЕ РАСПРОСТРАНЕНИЯ УПРУГИХ ВОЛН В ТРЕЩИНОВАТЫХ И ПОРИСТЫХ СРЕДАХ
}

\author{
Михайленко Б.Г., Мартынов В.Н., Фатьянов А.Г.
}

(Институт вычислительной матежатики и матезяатической геофизики СО РАН)

Представлены результаты численного моделирования распространения упругих волн в различных моделях пористыг и анизотропных сред. Для случая пористых сред используется алгоритм, основанньй на применении интегрального преобразования Фурье-Бесселя по пространственной переменной и преобразования Фурье по времени, в результате исходная задача для уравнений Френкеля-Био сводится к серии одномерньх задач для решения которых используется матричный формализм. Это позволяет в матричном виде свести искомую постановку к краевой задаче для обыкновенных дифференциальных уравнений, что, в свою очередь, дает возможкность развить эффективный аналитический алгоритм расчёта пористых волновых полей для произвольных плоско слоистых сред содержащих пористые слои [1].

Численное моделирование процессов распространения сейсмических волн в сложно построенных упругих анизотропных средах, произвольного вида симметрии, проводится на основе полной системы уравнений теории упругости записанной в скоростях перемещениях и напряжений с соответствующими граничными и начальными условиями. Для решения данной задачи используется метод конеqных интегральных преобразований по пространственным горизонтальным переменным и применение конечно разностного метода для репения редучированной задачи [2], [3]. Использование данного метода позволяет разработать алгоритм для 3-х мерного численного моделирования процессов распространения упругих волн в анизотропных неоднородных средах. Важной особенностью данного алгоритма является возможность их эффективной программной реализация на многопроцессорных вычислительных машинах.

На основе данного алгоритма разработан программный комплекс для проведения численного моделирования распространения упругих волн в реальных геофизических моделях анизотропных сред содержащих трещиноватые слои. Предполагается, что трещины имеют преимущественную ориентацию. На основе методов объемного осреднения с учетом свойств скелета, плотности трещин в единице объема, степени их заполнения флюидом, а также коэффициента распора трещин могут быть рассчитаны модули угтугости для анизотропной модели среды. Результаты численного моделирования могут быть использованы для проведения вычислительных экспериментов моделирующих типичные ситуация возникающие при мониторинге продуктивных горизонтов.

С целью исследования влияние анизотропии и трещиноватости на волновое поле проведена серия расчетов моделируюших распространения упругих волн, возникающих от сосредоточенньх истояников, расположенных в вертикально неоднородных анизотропных моделях характерных для реальных геофизических сред. Рассмотрены случаи формирования волнового поля для моделей сред, включаюощих тонкие анизотропные слои с различтыми коэффициентами анизотропии. При исследовании трещиноватых упругих сред и для разработки алгоритмов и программного обеспечения, ориентированного на выявление трещиноватости, необходимо, во-первых, понимать влияние этой трециноватости на волновое поле и, во-вторых, иметь возможность моделировать такое волновое поле с целью опробования новых алгоритмов обработки сейсмических данных.

Показано, что присутствие в моделях сред анизотропных слоев, у которьх ось ориентации трансверсально изотропной среды расположена под углам к границе раздела, приводит к существенным амплитудным и фазовым изменением в отраженных и проходящих упругих волнах, которых могут служить признаками наличия анизотропии или трещиноватости в модели среды.

Результаты численного моделирования могут быть использованы для развития физико-геологических основ сейсмического метода, а также для тестирования существующих систем обработки геофизической информации и создания новых.

Работа выполнена в рамках НИР СО РАН иГд-ИВМиМГ 2/2007

ЛИТЕРАТУРА

1. Мирошников В.В., Фатьянов А.Г. Полуаналитический метод расчёта волновых полей в слоистых пористых средах // Математическое моделирование в геофизике. Новосибирск, 1993. - с. 27 58 .

2. Martynov, V.N., and Mikhailenko, B.G. (1988), Calculation of Complete Theoretical Seismograms for Anisotropic Media. Inverse Modeling in Exploration Geophysics\}, Proceedings of the 6th International Mathematical Geophysics Seminar held at the Free University of Berlin, Feb. 3--6, 323--348.

3. Алексеев Ю. А. Мартынов В. Н. Михайленко Б. Г. (2004), Моделирование распространения упрутих вотн в трешиноватых средах, Труды Международной конференции по вычнслительной математике Новосибирск, 377-384. 